\title{
Aberrant expression of B203.13 antigen in acute lymphoid leukemia of $B$-cell origin
}

\author{
GIULIANA GOBBI $^{1}$, PRISCO MIRANDOLA ${ }^{1}$, CHIARA MALINVERNO $^{1}$, IVONNE SPONZILLI ${ }^{1}$, \\ CECILIA CARUBBI $^{1}$, FRANCESCA RICCI ${ }^{1}$, ROBERTO BINAZZI ${ }^{2}$, GIUSEPPE BASSO ${ }^{3}$, \\ GABRIELLA GIULIANI-PICCARI ${ }^{4}$, GIULIA RAMAZZOTTI ${ }^{4}$, GUIDO PASQUANTONIO ${ }^{5}$, \\ LUCIO $\mathrm{COCCO}^{4}$ and MARCO VITALE ${ }^{1}$
}

\author{
${ }^{1}$ Department of Anatomy, Pharmacology and Forensic Medicine, Human Anatomy Section, University of Parma, \\ Ospedale Maggiore, Via Gramsci 14, I-43100, Parma; ${ }^{2}$ I.R.C.C.S., Galeazzi Orthopaedic Institute, I-20161 \\ Milano; ${ }^{3}$ AIEOP Reference Laboratory for Immunophenotypic Studies, Department of Pediatrics, \\ University of Padua, Via Giustiniani 3, I-35128 Padua; ${ }^{4}$ Cellular Signalling Laboratory, Department \\ of Anatomical Sciences, University of Bologna, Via Irnerio 48, I-40126 Bologna; \\ ${ }^{5}$ Department of Dental Sciences, University of Tor Vergata, Rome, Italy
}

Received February 12, 2008; Accepted March 11, 2008

DOI: 10.3892/ijo_00000017

\begin{abstract}
The B203.13 monoclonal antibody was developed by immunizing mice with the $\mathrm{B} /$ monocyte biphenotypic cell line B1b. During normal hematopoiesis B203.13 is expressed on a fraction of $\mathrm{CD}_{3} 4^{+}$cells, while on mature cells it is only present on B-lymphocytes. We tested this antibody as a marker of childhood B-acute lymphoblastic leukemia (B-ALL). Bone marrow aspirates from 139 cases of early B-ALL and 25 controls were studied. About $40 \%$ of the B-ALL patients expressed B203.13. In these patients, B203.13 was constantly co-expressed with CD10, but never co-expressed with CD20, contrary to the controls. The $\mathrm{CD} 10^{+} / \mathrm{B} 203.13^{+}$phenotype was specific to $\mathrm{B}-\mathrm{ALL}$, since $\mathrm{CD} 10^{+} / \mathrm{CD} 20^{+}$cells from common acute lymphoblastic leukemia (c-ALL) did not express B203.13. We concluded that the use of B203.13 in association with CD10 and CD20 provides meaningful information for distinguishing normal residual B-cells from leukemic B-lymphoblasts and that recurrence of a CD10+/ B203.13+ phenotype after transplantation may be a very early relapse indicator of early B-acute lymphoblastic leukemia.
\end{abstract}

\section{Introduction}

Acute leukemia has long been recognized as a heterogeneous disease. To the classical morphological distinction between acute lymphoblastic and myeloblastic leukemia, a wide array

Correspondence to: Professor Lucio Cocco, Cellular Signalling Laboratory, Department of Anatomical Sciences, University of Bologna, Via Irnerio 48, I-40126 Bologna, Italy

E-mail:1ucio.cocco@unibo.it

Key words: B203.13, B-acute lymphoblastic leukemia, childhood leukemia phenotype of disease subsets has been added. These new differentiations are based on immunophenotype and, more recently, on gene expression profiling (1). Additionally, differential chromosomal and gene abnormalities in leukemia, which may be reflected in immunophenotypical alterations, define biological subsets of the disease with therapeutical implications and prognostic importance (2). For instance, using microarray expression profiling, B-acute lymphoblastic leukemia (B-ALL) carrying an MLL gene rearrangement was separated from other B-ALL (3); similarly, several known leukemia classes have been described on the basis of gene expression profiles, with new classes being identified (4). Moreover, a strong prognostic significance for at least 14 independent proliferation-related genes in the treatment outcome of childhood ALL has recently been demonstrated (5).

Most genetic studies, however, demonstrate that gene coding for known diagnostic marker proteins routinely monitored in B-ALL immunophenotyping are among the best transcripts for discrimination between leukemia subclasses (6). This strong correlation between the diagnostic value of gene expression profiling and that of immunophenotyping, reinforces the use of flow cytometry detection of leukemiaassociated antigens as a core diagnostic tool for leukemia diagnosis (7). The classical opinion that acute lymphoblastic leukemia developed from normal hematopoietic cells blocked at a particular differentiation stage, has recently been re-evaluated by the demonstration that leukemic cells often display immunophenotypic features which are usually undetectable in normal precursor cells, the so-called aberrant phenotype. These leukemia-associated phenotypes often involve cross-lineage antigen expression, asynchronous antigen expression, or variations in the antigen expression levels (8-11). In this regard, emphasis has increasingly been placed on the identification of new monoclonal antibodies highly capable of discriminating between leukemic and normal cells (12). Recently we described the expression pattern of the monoclonal antibody (MoAb) B203.13, that we 
originally generated by immunizing mice with the B myeloid biphenotypic human cell line B1b $(13,14)$. The antigen recognized by B203.13 is expressed i) in most peripheral adult and cord blood B-cells and ii) at minimal density in mature monocytes, while iii) it is undetectable in granulocytes, T and NK cells, and erythrocytes. In both cord blood and adult bone marrow, B203.13 MoAb recognizes a surface marker present on the progenitor cells of several hematopoietic lineages, being transiently expressed on $\mathrm{CD} 71^{+}$early erythroid, $\mathrm{CD}_{3} 3^{+}$early myeloid and $\mathrm{CD}^{+}$early $\mathrm{T}-$ lymphoid precursors. B203.13 is also expressed on early CD34 ${ }^{+} / 19^{+}$ B-lymphoid precursors. We also demonstrated that the CD34+/B203.13+ subset of human hematopoietic progenitors is significantly enriched in megakaryocyte progenitors (CFUmeg) (13). Although not all individuals express detectable levels of B203.13, its distribution is distinguished from other known surface molecules, specifically from the polymorphic HLA-DR and CD1 family of proteins (14).

Given the broad expression of B203.13 on hematopoietic precursors and its selective persistence in the B-cell lineage, we were prompted to study the expression of B203.13 in the bone marrow of young B-ALL patients, for its potential applications in acute leukemia subsetting and as an early marker of disease relapse.

\section{Materials and methods}

Patients. One hundred and thirty-nine de novo early B-ALL and 25 pediatric patients affected by non-clonal hematopoietic disorders were included in this study. The cohort of B-ALL patients comprised 87 males and 52 females, with a median age of 9.4 years (range 1-21 years). In all cases, ALL diagnosis was performed by morphology, cytochemistry, cytogenetics and molecular genetics. The patients affected by ALL included in this study derived from a single institution and were enrolled in the AIEOP 95-2000 protocol according to the diagnostic guidelines. The morphological classification was performed by three independent investigators, and a conclusive diagnosis for every case was reported according to the FAB criteria.

Immunophenotypic analysis. Immunophenotypic analyses were performed by multiparameter three- or four-color flow cytometry using an Epics XL flow cytometer (Beckmann Coulter, Inc., Miami, FL, USA). Data were acquired using Expo32 software (Beckmann Coulter). ALL diagnosis was made using the fluorochrome-conjugated MoAb panel routinely employed by the AIEOP Reference Laboratory for Immunophenotypic Studies of the University Hospital of Padua. Briefly, at least $0.5 \times 10^{6}$ cells in a final volume of $100 \mu 1$ were used in each analysis and MoAbs, directly labelled with fluorescein isothiocyanate (FITC), phycoerythrin (PE), or with the fluorochrome tandem conjugates PE-Texas red (ECD) or PE-cyanine 5 (PECy5), were combined in each tube according to previously reported methods $(15,16)$. Isotype-matched negative controls were used for each MoAb combination.

The following MoAbs were used: CD2, 3, 10, 13, 14, 16, $19,22,24,33,38,58,66 \mathrm{c}$ and 135, NG2 and TdT (Beckmann Coulter), CD5, 8, 11a, 15, 20, 34, and 56 (Becton Dickinson,
San José, CA, USA), CD4, 7, 45, and 65, and HLADR (Caltag Laboratories, San Francisco, CA, USA), and CD133 (Miltenyi, Bergisch Gladbach, Germany) (17). FITC-conjugated B203.13 MoAb was added to the panel of markers described above. The instrument setup and fluorescence compensation were optimized daily at the beginning of each procedure by analyzing the fluorescence of normal T-lymphocytes (always present in the samples) labeled with the anti-CD4FITC/ CD8PE/CD3ECD/CD45PECy5 four-color combination (6).

An immunological gate including all blast cells was set in all tube combinations based on the positive expression of CD19. Specifically, a side scatter dot plot (SSC; logarithmic amplification) vs. CD19 was used to design the immunological gate. CD19, instead of the more commonly used CD45 (18), was chosen for gating since CD45 expression can be low or negative in a large proportion of B-cell origin ALL. By using the CD19/log SSC gating strategy, all blast cells were included, while the percentage of normal B-cells included in the gate was not significant. In fact, the presence of normal B-cells was always evaluated using the CD19/34/45 combination: the percentage of CD19 positive cells with normal expression of CD45 and negative for CD34 was constantly lower than $5 \%$ in the gated population $(15,16)$. The CD10/ $19 / 20 / 34$ combination was chosen to identify the expression of B203.13 in the B-cell lineage.

\section{Results and Discussion}

It is now well established that immunophenotypic analysis of neoplastic cells is an invaluable tool for the accurate diagnosis and classification of acute leukemias, as well as for the detection of minimal residual disease (MRD) after therapy and for the early diagnosis of relapse after bone marrow transplantation. This is essentially due to the fact that flow cytometry immunophenotyping is more sensitive than morphology in detecting leukemic cells and is applicable to a high proportion of leukemias.

Although prognosis in paediatric acute lymphoblastic leukemias can be assessed quite accurately by PCR and transcriptional profiling, a close correlation between molecular methods and flow cytometric immunophenotyping in the assessment of leukemia subsets and subsequent MRD and therapy monitoring, has recently been demonstrated $(2,19,20)$. Therefore, emphasis is increasingly placed on the identification of new phenotypic markers that might be relevant for optimizing discrimination between leukemic and normal cells, and, perhaps more importantly, for MRD detection and the post-transplantation monitoring of relapse occurrence.

Recently, we demonstrated that B203.13 was expressed on $\mathrm{CD}_{3} 4^{+}$early myeloerythroid committed progenitors and on a subset of CD34- cells that included immature progenitors of most hematopoietic lineages $(13,14)$. B203.13 was also expressed on committed $\mathrm{CD} 34^{+} / \mathrm{CD} 19^{+}$precursors, while it was rapidly down-modulated during further hematopoietic differentiation. In mature cells it was expressed on B-lymphocytes in $\sim 40-50 \%$ of healthy individuals. Moreover, it was recently demonstrated that the expression of CD20 or CD10 in B-ALL has a prognostic significance in relation to age (9-11). 


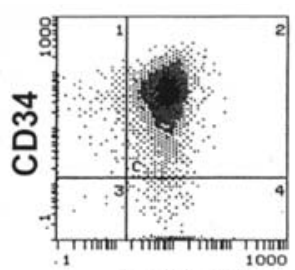

B203.13

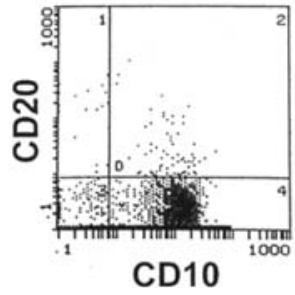

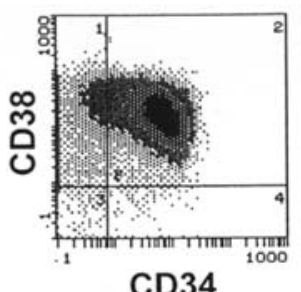

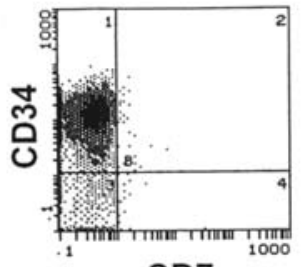

CD7
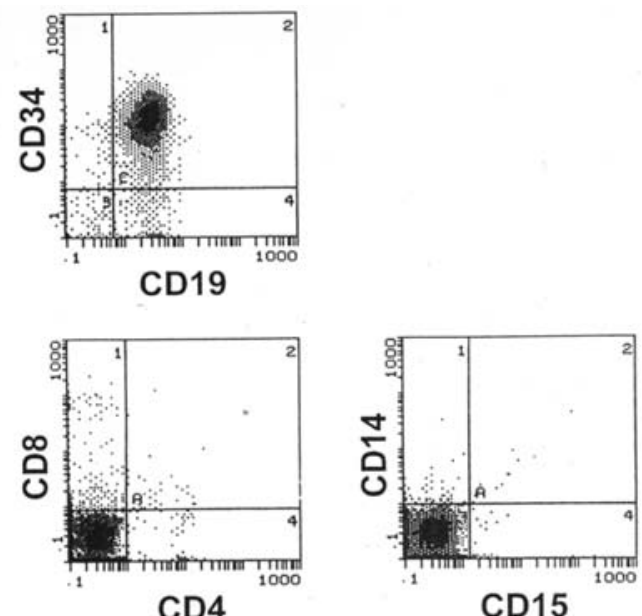

Figure 1 . Bone marrow flow cytometry immunophenotype of a B203.13+ B-ALL. Virtually all cells were $\mathrm{CD}^{+} 4^{+} / \mathrm{B} 203.13^{+} / \mathrm{CD} 19^{+} / \mathrm{CD} 10^{+}$, while T-cell markers like CD7, 4 and 8, and myelomonocytic CD15 and 14 markers, were all negative. CD20 was also always negative. Representative of 54 subjects.
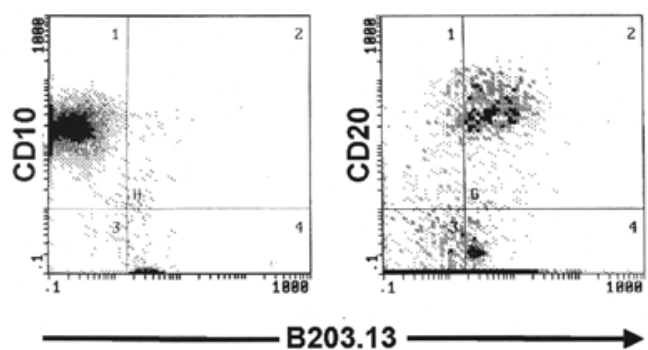

Figure 2. Flow cytometry analysis of B203.13 expression in normal bone marrow CD19-gated cells. B203.13 is expressed in mature CD10/CD20+ $\mathrm{B}$-cells, while it is not expressed in $\mathrm{CD} 10^{+} / \mathrm{CD} 20^{-} \mathrm{B}$-cell progenitors. Representative of 11 subjects.
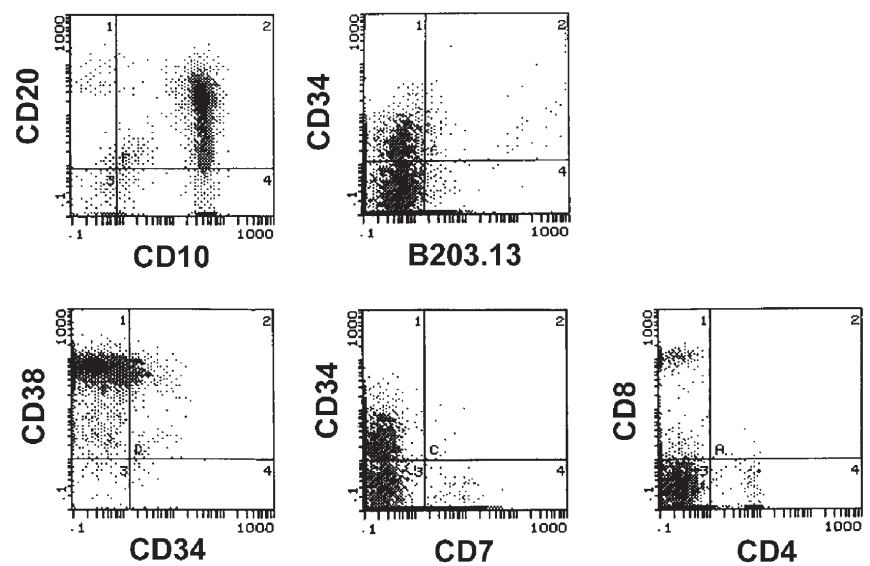

Figure 3. Flow cytometry analysis of B203.13 expression in a case of c-ALL. CD10+/CD20+ leukemic cells did not express B203.13. Representative of $5 \mathrm{c}$-ALL patients.

To evaluate B203.13 expression in B-lineage ALL and to compare the results with normal bone marrow, we tested this new monoclonal antibody in 139 early B-ALL at diagnosis stage and in 25 samples collected from children undergoing bone marrow aspiration for non-lymphoproliferative

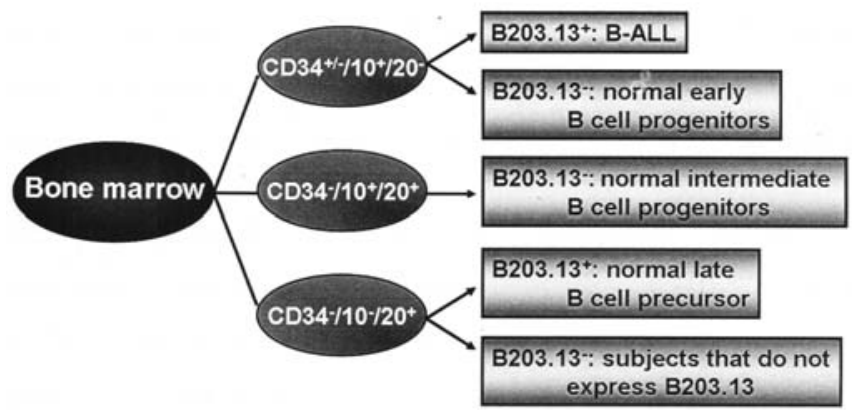

Figure 4. Proposed scheme of B203.13 expression in the bone marrow of normal or B-ALL patients. Identification of a leukemic phenotype in combination with CD10 and 20.

disorders (controls). Fifty-four out of 139 early-B-ALL patients (38.8\%) were positive for B203.13. In these patients, CD19+ bone marrow (BM) cells constantly co-expressed B203.13 and CD10; whereas B203.13 was never coexpressed with CD20 (Fig. 1).

Normal bone marrow, on the contrary, showed an opposite expression pattern: B203.13 was never co-expressed with CD10, while it was constantly co-expressed with CD20 (11 out of 25 controls expressed B203.13) (Fig. 2). To exclude that the co-expression of B203.13 with CD10 might be a general feature of acute leukemias, rather than a specific B-ALL phenotype, we tested five cases of $\mathrm{CD} 10^{+} / \mathrm{CD} 20^{+}$common acute lymphoblastic leukemia (c-ALL), and all resulted negative for B203.13 (Fig. 3).

As a whole, these data suggest that normal B-lymphocytes express B203.13 in the early stages of development (with a phenotype that is $\mathrm{CD} 19^{+} / \mathrm{CD} 34^{+} / \mathrm{CD} 10^{-/ B} 203.13^{+}$); the expression of B203.13 is then down-regulated at the pre-B-cell stage $\left(\mathrm{CD} 19^{+} / \mathrm{CD} 34^{+/ / C D} 10^{+/ C D} 20^{-/ B} 203.13^{-}\right.$; CD19+/CD34-CD10+/CD20+/B203.13-), and finally re-expressed with $\mathrm{CD} 20$ in mature B-cells $\left(\mathrm{CD} 19^{+} / \mathrm{CD} 34 / \mathrm{CD} 10^{-} /\right.$ $\left.\mathrm{CD} 20^{+} / \mathrm{B} 203.13^{+}\right)$. On the contrary, the co-expression of B203.13 with CD10 appears to be a phenotypic characteristic 
of B-ALL. The proposed B203.13 expression pattern in normal vs. B-ALL BM cells is summarized in Fig. 4.

The use of B203.13 in association with CD10 and CD20 provides meaningful information to distinguish normal residual B-cell from leukemic B-lymphoblasts. Thus, in $\mathrm{B} 203.13^{+}$ALL it is conceivable that the recurrence of a $\mathrm{CD} 10^{+} / \mathrm{B} 203.13^{+}$phenotype after bone marrow transplantation may be a very early relapse indicator.

\section{Acknowledgements}

This work was supported by AIL grants to MV, and MIUR PRIN 2006 to GG. We are grateful to Instrumentation Laboratory, Italy, and to Domenico Manfredi and Luciana Cerasuolo for technical and editorial support.

\section{References}

1. Downing JR and Shannon KM: Acute leukemia: a pediatric perspective. Cancer Cell 2: 437-445, 2002.

2. Mussolin L, Pillon M, Conter V, et al: Prognostic role of minimal residual disease in mature B-cell acute lymphoblastic leukemia of childhood. J Clin Oncol 25: 5254-5261, 2007.

3. Armstrong SA, Staunton JE, Silverman LB, et al: MLL translocations specify a distinct gene expression profile that distinguishes a unique leukemia. Nat Genet 30: 41-47, 2002.

4. Yeoh EJ, Ross ME, Shurtleff SA, et al: Classification, subtype discovery, and prediction of outcome in pediatric acute lymphoblastic leukemia by gene expression profiling. Cancer Cell 1: 133-143, 2002.

5. Flotho C, Coustan-Smith E, Pei D, et al: A set of genes that regulate cell proliferation predicts treatment outcome in childhood acute lymphoblastic leukemia. Blood 110: 1271-1277, 2007.

6. De Zen L, Bicciato S, te Kronnie G, et al: Computational analysis of flow-cytometry antigen expression profiles in childhood acute lymphoblastic leukemia: an MLL/AF4 identification. Leukemia 17: 1557-1565, 2003.

7. Coustan-Smith E, Ribeiro RC, Stow P, et al: A simplified flow cytometric assay identifies children with acute lymphoblastic leukemia who have a superior clinical outcome. Blood 108: 97-102, 2006.
8. Vidriales MB, San-Miguel JF, Orfao A, et al: Minimal residual disease monitoring by flow cytometry. Best Pract Res Clin Haematol 16: 599-612, 2003.

9. Gleissner B, Goekbuget N, Rieder $\mathrm{H}$, et al: $\mathrm{CD} 10^{-}$pre-B acute lymphoblastic leukemia (ALL) is a distinct high-risk subgroup of adult ALL associated with a high frequency of MLL aberrations: results of the German Multicenter Trials for Adult ALL (GMALL). Blood 106: 4054-4056, 2005.

10. Hilden JM, Dinndorf PA, Meerbaum SO, et al: Analysis of prognostic factors of acute lymphoblastic leukemia in infants: report on CCG 1953 from the Children's Oncology Group. Blood 108: 441-451, 2006.

11. Jeha S, Behm F, Pei D, et al: Prognostic significance of CD20 expression in childhood B-cell precursor acute lymphoblastic leukemia. Blood 108: 3302-3304, 2006.

12. Chen JS, Coustan-Smith E, Suzuki T, et al: Identification of novel markers for monitoring minimal residual disease in acute lymphoblastic leukemia. Blood 97: 2115-2120, 2001.

13. Vitale M, Zauli G, Bassini A, et al: Selective enrichment in human megakaryocyte progenitors by the B203.13 surface differentiation antigen. Br J Haematol 99: 766-769, 1997.

14. Zamai L, Vitale M, Bennett IM, et al: A novel surface marker (B203.13) of human haemopoietic progenitors, preferentially expressed along the $\mathrm{B}$ and myeloid lineages. Br J Haematol 102: 965-975, 1998.

15. Basso G, Buldini B, De Zen L, et al: New methodologic approaches for immunophenotyping acute leukemias. Haematologica 86: 675-692, 2001.

16. De Zen L, Orfao A, Cazzaniga G, et al: Quantitative multiparametric immunophenotyping in acute lymphoblastic leukemia: correlation with specific genotype. I. ETV6/AML1 ALLs identification. Leukemia 14: 1225-1231, 2000.

17. Veltroni M, De Zen L, Sanzari MC, et al: Expression of CD58 in normal, regenerating and leukemic bone marrow B cells: implications for the detection of minimal residual disease in acute lymphocytic leukemia. Haematologica 88: 1245-1252, 2003.

18. Braylan RC, Atwater SK, Diamond L, et al: U.S.-Canadian Consensus recommendations on the immunophenotypic analysis of hematologic neoplasia by flow cytometry: data reporting. Cytometry 30: 245-248, 1997.

19. Bene MC: Immunophenotyping of acute leukaemias. Immunol Lett 98: 9-21, 2005.

20. Haferlach T, Kern W, Schnittger S, et al: Modern diagnostics in acute leukemias. Crit Rev Oncol Hematol 56: 223-234, 2005. 